\title{
ISBN Group Agencies
}

\author{
(Country / Group No. / Address of ISBN Group Agency)
}

\author{
Afghanistan / 978-9936 \\ Registration, Copyright and ISBN \\ Ministry of Information and Culture \\ Mohamad Jan Khan watt beside Spin zar \\ hotel \\ Kabul \\ Afghanistan \\ Tel (+93) 202101312 \\ Tel (+93) 794899224 \\ E-mail:moic.isbn@gmail.com \\ Albania / 978-9928 + 978-99927 + 978- \\ $99943+978-99956$ \\ Biblioteka Kombetare \\ National Agency of Standard International \\ Numbers of (ISBN ,ISSN, ISMN) \\ Sheshi Skënderbe \\ Tirana \\ Albania \\ Tel +355 694094448 \\ E-mail: Enkelejda.Gashi@bksh.al \\ URL http://www.bksh.al
}

Algeria / 978-9931 + 978-9947 + 978-9961

Agence algérienne de l'ISBN

Bibliothèque Nationale d'Algérie

BP. 127, El Hamma

El Annasser, Alger

Algeria

Tel (+213 (0) 21675760

Fax (+213 (0) 21671867

E-mail: hgounni2013@yahoo.fr

URL http://www.biblionat_algerie.dz

Andorra / 978-99913 + 978-99920

Andorran Standard Book Numbering Agency

National Library of Andorra

Placeta de Sant Esteve $\mathbf{s} / \mathrm{n}$

Andorra la Vella AD500

Andorra

Tel (+376) 826445

Fax (+376) 829445

E-mail: bncultura.gov@andorra.ad

URL http://www.bibliotecanacional.ad

Argentina / 978-950 + 978-987

Cámara Argentina del Libro

Av. Belgrano 15804 Piso

C1093AAQ Buenos Aires

Argentina

Tel (+54 11) 43818383

Tel (+54 11) 5353 1265/63

Fax (+54 11) 53531270

E-mail: registrolibros@editores.org.ar

URL http://www.isbnargentina.org.ar
Armenia / 978-5 + 978-9939 + 978-99930 + 978-99941

National Book Chamber of Armenia

ISBN Agency

G. Kochar St. 21

0009 Yerevan

Armenia

Tel (+ 374 10) 527595
Tel (+ 374 10) 582511
E-mail: grapalat@arminco.com
URL http://www.book-chamber.am

Australia / 978-0 + 978-1

ISBN Agency - Thorpe Bowker

Level One, 607 St Kilda Road

Melbourne

Victoria, 3004

Australia

$$
\begin{aligned}
& \text { Tel (+61 3) } 85178349 \\
& \text { Fax (+61 3) } 85178399 \\
& \text { E-mail: isbn@thorpe.com.au } \\
& \text { URL http://www.myidentifiers.com.au }
\end{aligned}
$$

Austria / 978-3

Hauptverband des Österreichischen Buchhandels

Grünangergasse 4

1010 Wien

Austria

$$
\begin{aligned}
& \text { Tel (+43 1) } 5121535 \text { (14) } \\
& \text { Fax (+43 1) } 5128482 \\
& \text { E-mail: isbn@hvb.at } \\
& \text { URL http://www.isbn.co.at }
\end{aligned}
$$

Azerbaijan / 978-5 + 978-9952

ISBN Agency

Khazar University Press

11, Mahsati St., Nizami District

Baku AZ1096

Azerbaijan

Tel (+994 12) 4217927

Fax (+994 12) 4989379

E-mail: Gmehdiyeva@khazar.org

URL http://www.khazar.org

Bahrain / 978-99901 + 978-99958

Information Affairs and Authority

Publication and Press

P.O. BOX: 253

Isa Town

Bahrain

Tel (+973) 17372836

Fax (+973) 17372220

E-mail: batool@iaa.gov.bh
URL http://www.moci.gov.bh/ar/ Header/s,2827

Bangladesh / 978-984

National Library of Bangladesh, Directorate of Archives \& Libraries, ISBN agency

32, Justice S. M. Morshed Sarani

Sher-e-Bangla Nagar (Agargaon)

Dhaka 1207

Bangladesh
Tel (+880 2) 9129992
Fax (+880 2) 9118704
Fax (+880 2) 9135709
E-mail:nanldirector@gmail.com
URL http://www.nanl.gov.bd

Belarus / 978-5 + 978-985

National Book Chamber of the Belarus, ISBN Agency

31a Very Khoruzhey St.

220002 Minsk

Belarus

Tel (+375) 172886715

Fax (+375) 172832960

E-mail:Palata@natbook.org.by

URL http://natbook.org.by

Belgium - French speaking / 978-2

AFNIL

35, rue Grégoire de Tours

75006 Paris

France

Tel (+33 1) 44412919

Fax (+33 1) 44412903

E-mail:afnil@electre.com

URL http://www.afnil.org

Benin / 978-99919

Agence Nationale ISBN, Bibliothèque Nationale

01 BP 401

Porto-Novo

Benin

Tel (+229) 20246585

Fax (+229) 20246585

E-mail: madego1@yahoo.fr

Bermuda / 978-0 + 978-1

ISBN Agency of Bermuda

Bermuda National Library

Par-la-Ville

13 Queen Street

Hamilton HM11

Bermuda 
Tel (1 441) 296-1297 ext. 229

Tel (1 441) 296-1297 ext 223

Fax (1 441) 2928443

E-mail: ejhollis@gov.bm

URL http://www.bnl.bm

Bhutan / 978-99936

ISBN Agency, The Centre for Bhutan Studies

Post Box 1111

Lanjophaka

Thimphu

Bhutan

Tel (+975 2) 321005

Tel (+975 2) 321111

Fax (+975 2) 321001

E-mail:cbs@druknet.bt

Bolivia, Plurinational State of / 978-99905 + 978-99954 + 978-99974

Agencia ISBN, Cámara Boliviana del Libro

Calle Capitán Ravelo No. 2116

Casilla 682

La Paz

Bolivia, Plurinational State of

Tel (+591 2) 2113264

Tel (+591 2) 2444239

Fax (+591 2) 441523

E-mail: isbn@camaralibrolapaz.org.bo URL www.camaralibrolapaz.org.bo

Bosnia and Herzegovina / 978-86 + 978$9926+978-9958$

ISBN Centre, National and University Library of Bosnia and Herzegovina

Zmaja od Bosne 8b

71000 Sarajevo

Bosnia and Herzegovina

Tel (+387 33) 212435

Fax (+387 33) 212435

E-mail: isbn-bih@nub.ba

URL http://www.nub.ba

Botswana / 978-99912 + 978-99968

Botswana National Library Service

National Reference Library

P/ Bag 0036

Gaborone

Botswana

Tel (+267) 3952397

Fax (+267) 3901149

E-mail: mtamocha@gov.bw

Brazil / 978-85

Brazilian ISBN Agency

National Library Foundation - Brazil

Rua Debret 23 sala 803 Centro

Rio de Janeiro - RJ CEP: 20030-080

Brazil

Tel (55 21) 3095.3953

Tel (55 21) 2220.1701
Fax (55 21) 2544.8596

Fax (55 21) 2220.1702

E-mail: isbn@bn.br

URL http://www.isbn.bn.br

Brunei Darussalam / 978-99917

Pusat Kebangsaan ISBN, Literature Bureau Library Section, Brunei

Perpustakaan

Dewan Bahasa Dan Pustaka

Jalan Elizabeth II

Bandar Seri Begawan, BS 8711

Brunei Darussalam

Tel (+673 2) 235501

Tel (+673 2) 222135

Fax (+673 2) 224763

E-mail: kb_perpustakaan@brunet.bn

URL http://www.dplibrary.gov.bn

Bulgaria / 978-619 + 978-954

National ISBN Agency, National Library St. Cyril and St. Methodius

Boulevard Vasil Levski 88

1037 Sofia

Bulgaria

Tel (+359 2) 9461143

Tel (+359 2) 9183219

Fax (+359 2) 8435495

E-mail: isbn@nationallibrary.bg

URL http://www.nationallibrary.bg

Cambodia / 978-99950 + 978-99963

ISBN Agency, National Library of Cambodia Christopher Howes Street 92

Daun Penh District

Phnom Penh

Cambodia

$$
\begin{aligned}
& \text { Tel (+855) (0) } 11303701 \\
& \text { Tel (+855) (0) } 23430609 \\
& \text { Fax +855 (0) } 23430609 \\
& \text { E-mail: mony@bnc-nlc.info } \\
& \text { URL http://www.bnc-nlc.info }
\end{aligned}
$$

\section{Cameroon / 978-9956}

Agence ISBN, Bibliothèque Nationale

Direction du Livre et de la Lecture

Ministère des Arts et de la Culture, BP 1053

Yaoundé

Cameroon

$$
\begin{aligned}
& \text { Tel (+237) } 22237001 \\
& \text { Tel (+237) 22 22 } 4785 \\
& \text { Fax (+237) } 22226579
\end{aligned}
$$

E-mail:dbplminac@yahoo.fr

Canada - English Language / 978-0 + 978-1 Canadian ISBN Agency, Library and Archives Canada / Bibliothèque et Archives Canada

Library and Archives Canada / Bibliothèque et Archives Canada
395 Wellington Street

Ottawa

ON K1A 0N4

Canada

Tel (+1 819) 9946872

Tel (+1 866) 5787777 (toll free)

Fax (+1 819) 9346777

E-mail: isbn@bac-lac.gc.ca

URL http://www.collectionscanada. gc.ca

Canada - French Language / 978-2

Bibliothèque et Archives nationales du Québec

Agence ISBN

2275, rue Holt

Montréal (Québec)

H2G 3H1

Canada

Tel (+1 514) $8731101 \times 3785$

Fax (+1 514) 8734310

E-mail: isbn@banq.qc.ca

URL http://www.banq.qc.ca

Caribbean Community / 978-976

Caribbean Regional ISBN Agency

Caribbean Community Secretariat

PO Box 10827, Turkeyen

Greater Georgetown

Guyana

Tel (+592) 222 0001-075

Fax (+592) 222 - 0170

E-mail: doccentre@caricom.org

URL http://www.caricom.org

Chile / 978-956

Agencia Chilena ISBN

Cámara Chilena del Libro A. G.

Avda. Libertador Bernardo O'Higgins 1370, Oficina 502

Santiago de Chile

Chile

Tel (+56 2) 6724088

Tel (+56 2) 6989519

Fax (+56 2) 6874271

E-mail: isbnchile@tie.cl

China / 978-7

China ISBN Agency (Barcode Center of GAPP)

10 Xianxiao Hutong, Room 603

Dongcheng District

100005, Beijing

China

Tel (+86 10) 58689870

Fax (+86 10) 58689995

E-mail: Chinaisbn@126.com

URL http://www.capub.cn/shtmsl/ index_shtmsl.shtml?id=8 
Colombia / 978-958

Agencia Colombiana del ISBN

Cámara Colombiana del Libro

Calle 35 \# 5 A - 05, Barrio La Merced

Bogotá D.C.

Colombia

$$
\begin{aligned}
& \text { Tel (+57 1) } 3230111 \times 108 \\
& \text { Fax (+57 1) } 2858023 \\
& \text { E-mail: agenciaisbn@camlibro.com.co } \\
& \text { URL http://www.camlibro.com.co }
\end{aligned}
$$

\section{Congo, The Democratic Republic of the /} 978-99951

Bibliothèque Nationale du Congo

10, Bld Colonel Tshatshi, B.P.

5432 Kinshasa - Gombe

Congo, The Democratic Republic of the

Costa Rica / 978-9930 + 978-9968 + 978-9977

Biblioteca Nacional, Departamento Unidad Técnica

Calles 15-17, Av. 3 y 3b Primer Piso

Edificio anexo a la Biblioteca Nacional

Apdo. 10.008-1000 San José

Costa Rica

Tel $(+506)$ 2223-1303

Tel (+506) 2233-1706

Fax (+506) 22235510

Fax (+506) 2233-1706

E-mail: isbn@sinabi.go.cr

URL http://www.sinabi.go.cr

Croatia / 978-86 + 978-953

Hrvatski ured za ISBN

Nacionalna i sveučilišna knjižnica u Zagrebu Ulica Hrvatske bratske zajednice 4

HR-10000 Zagreb

Croatia

Tel (+385 1) 6164288

Fax (+385 1) 6164191

E-mail: isbn@nsk.hr

URL http://www.nsk.hr/isbn

Cuba / 978-959

Cámara Cubana del Libro

Calle 15 No 602 esq. C

Vedado, Ciudad Habana

Cuba

Tel (+53 7) 8328829

Tel (+53 7) 8329526

Fax (+53 7) 333441

E-mail: isbn@cubarte.cult.cu

\section{Curaçao / 978-99904}

ISBN Agency

Bureau Intellectual Property

Berg Carmelweg 10A

Willemstad-Curaçao

Curaçao
Tel (+599 9) 4657800

Fax (+599 9) 4657692

E-mail:bipantil@curinfo.an

URL http://www.bip.an

Cyprus / 978-9963

Cyprus ISBN Registration Centre

The Cyprus Library

Eleftheria Sq.

1011 Nicosia

Cyprus

Tel (+357 22) 303180

Tel (+357 22) 818431

Fax (+357 22) 304532

E-mail: isbncentre48@yahoo.gr

URL http://www.cypruslibrary.gov.cy

Czech Republic / 978-80

Národni agentura ISBN v ČR

Národni knihovna České republiky

Národni agentura ISBN v ČR

Sodomkova 2/1146

10200 Praha 15

Czech Republic

Tel (+42 02) 221663306

E-mail: isbn@nkp.cz

URL http://www.nkp.cz

Denmark / 978-87

Dansk Biblioteks Center

Tempovej 7-11

DK 2750 Ballerup

Denmark

Tel (+45) 44867779
Fax (+45) 44867693
E-mail: ij@dbc.dk
URL http://www.isbn-kontoret.dk

Dominican Republic / 978-9945 + 978-99934

Agencia Domenicana de ISBN

Biblioteca Nacional Pedro Henríquez Ureña

Av. Cesar Nicolás Pensón \#91 Plaza de la Cultura

Santo Domingo, Distrito Nacional

Dominican Republic

Tel (+1) 8299462674

Fax (+1 809) 5637677

E-mail: greibbys_asencio@hotmail. com

URL http://www.bnrd.gov.do

Ecuador / 978-9942 + 978-9978

Agencia Ecuatoriana del ISBN

Av. Eloy Alfaro N29-61 e Inglaterra

Edif. Eloy Alfaro Piso $N^{\circ} 9$

Quito

Ecuador
Tel (+593 2) 2553311

Tel (+593 2) 2553314

Fax (+593 2) 2222150

E-mail: isbn@celibro.org.ec

URL http://www.celibro.org.ec

Egypt / 978-977

ISBN Agency

Head of Deposit Depart.

Egyptian National Library

Kornish el Nile, Ramelt Bolaq,

Cairo

Egypt

Tel (+20 2) 25750886

Tel (+20 2) 25751078

Fax (+20 2) 357589547

E-mail: darelkotob.fan@gmail.com

El Salvador / 978-99923 + 978-99961

Agencia salvadoreña del ISBN

Biblioteca Nacional de El Salvador "Francisco Gavidia", 4a

Calle Oriente \# 123

San Salvador

El Salvador

Tel (+503) 22812388

Tel (+503) 2221209922

Fax (+503) 2218847

E-mail: isbn@cultura.gob.sv

URL http://www.cultura.gob.sv

Eritrea / 978-99948

ISBN Agency Eritrea

Hdri Publishers

178 Tegadelti Street, House No. 35

P.O. Box 1081

Asmara

Eritrea

Tel (+2911) 126177

Fax (+2911) 125630

E-mail: hdripublishers@yahoo.com

Estonia / 978-5 + 978-9949 + 978-9985

Estonian ISBN Agency

National Library of Estonia

Tõnismägi 2

Tallinn 15189

Estonia

Tel (+372) 6307372

Fax (+372) 6311200

E-mail: eraamat@nlib.ee

URL http://www.nlib.ee/isbn

Ethiopia / 978-99944

Ethiopia ISBN Agency

National Archives and Library of Ethiopia

P.O.Box 717

Addis Ababa

Ethiopia 
Tel (+251) 0115530058

Fax (+251) 0115526411

E-mail: eskedar.girum@nala.gov.et URL http://www.nala.gov.et

European Union Organizations / 978-92 ISBN Agency

Publications Office, Business Development

\& Support Unit

ISBN/ISSN/DOI Agencies

2, rue Mercier

2985 Luxembourg

Luxembourg

Tel (+352) 2929-42830

Fax (+352) 2929-44440

E-mail: carol.riccalton@publications. europa.eu

URL http://www.publications.europa. eu

Faroe Islands / 978-99918 + 978-99972

Faroese ISBN office

Føroya Landsbókasavn

J. C. Svabosgøtu 16, P.O.B. 61

FR-110 Tórshavn

Faroe Islands

Tel (+298) 340525

E-mail: laurav@savn.fo

Finland / 978-951 + 978-952

Finnish ISBN Agency

National Library of Finland

P.O. Box 26 (Teollisuuskatu 23)

Fl-00014 University of Helsinki

Finland

Tel (+358 9) 19144329

Fax (+358 9) 19144341

E-mail: isbn-keskus@helsinki.fi

URL http://www.nationallibrary.fi/ index.html

France / 978-2 + 979-10

AFNIL

35, rue Grégoire de Tours

75006 Paris

France

Tel (+33 1) 44412919

Fax (+33 1) 44412903

E-mail: afnil@electre.com

URL http://www.afnil.org

Gabon (reserved) / 978-99902

Gabon (reserved)

N/A

Gabon

Gambia / 978-9983

Gambia ISBN Agency

The Gambia National Library Authority

R. G. Pye Lane
Banjul

Gambia

Tel (+220) 4226491

E-mail: national.library@qanet.gm

Georgia / 978-9941 + 978-99928 + 978-99940

ISBN Centre

National Parliamentary Library of Georgia

5/7 Gudiashvili st.

0107 Tbilisi

Georgia

Tel (+995 32) 2971632

Fax (+995 32) 2971615

E-mail:ninosim@hotmail.com

Germany / 978-3

ISBN-Agentur für die Bundesrepublik Deutschland

MVB Marketing- und Verlagsservice des Buchhandels $\mathrm{GmbH}$

Braubachstraße 16

D-60311 Frankfurt am Main

Germany

Tel (+49 69) 1306346

Fax (+49 69) 1306258

E-mail: isbn@mvb-online.de

URL http://www.german-isbn.de

Ghana / 978-9964 + 978-9988

Ghana Library Authority

George Padmore Research Library

on African Affairs

P.O. Box GP2970

Accra

Ghana

Tel (+233 30) 228402

Tel $(+23330) 247768$

Fax (+233 30) 247768

E-mail: padmoreresearch@ghla.org.gh

Gibraltar / 978-0 + 978-1

The ISBN Agency for Gibraltar

The John Mackintosh Hall

308 Main Street

Gibraltar

GX11 1AA

Gibraltar

Tel (+350) 20075669

Fax (+350) 20040843

E-mail:minculture@gibtelecom.net

Greece / 978-618 + 978-960

National Centre of ISBN Greece

National Library of Greece

Leof. Kavalas 31 and Spyrou Patsi 12

10447 Athens

Greece
Tel (+30 210) 3470330

Fax (+30 210) 3608141

E-mail: isbn@nlg.gr

URL http://www.nlg.gr/isbn

Guatemala / 978-9929 + 978-99922 +

978-99939

Agencia ISBN

Gremial de Editores de Guatemala

Ruta 6, 9-21 zona 4 nivel 8

Edificio Cámara de Industria

Ciudad Guatemala

Guatemala

Tel (+502) 23347942

Tel (+502) $23809000 \times 338$

Fax (+502) 23809110

E-mail: isbn@industriaguate.com

URL http://www.filgua.com

Haiti / 978-99935 + 978-99970

ISBN Agency

Bibliothèque Nationale d'Haïti

193, Rue du Centre

Port-au-Prince

Haiti

Tel (509) 2813-0236

E-mail: bibliothequenationaleht@ yahoo.fr

Honduras / 978-99926

Agencia ISBN de Honduras

Biblioteca Nacional

Barrio El Centro, Avda Cervantes

1 cuadra al Sur Hotel Prado

Tegucigalpa, M.D.C.

Honduras

Tel (+504) 2201746

Fax (+504) 2221335

E-mail: agenciasisbnhonduras@ hotmail.com

Hong Kong / 978-962 + 978-988

Books Registration Office

Hong Kong Public Libraries, Leisure and

Cultural Services Department

Room 805, Lai Chi Kok Government Offices

19 Lai Wan Road

Lai Chi Kok, Kowloon

Hong Kong

Tel (+852) 21809145

Tel (+852) 21809146

Fax (+852) 21809841

E-mail: bro@lcsd.gov.hk

URL http://www.hkpl.gov.hk/en/aboutus/services/book-registration/ isbn.html 
Hungary / 978-615 + 978-963

Magyar ISBN és ISMN Iroda

National Széchényi Library

Szent György tér 4-5-6

H-1014 Budapest

Hungary

Tel (+36 1) 2243840

Fax (+36 1) 2323530

E-mail: isbn@oszk.hu

URL http://www.oszk.hu

Iceland / 978-9935 + 978-9979

ISBN Agency Iceland

National and University Library of Iceland

Arngrímsgötu 3

IS-107 Reykjavík

Iceland

Tel (+354) 5255755

Fax (+354) 5255615

E-mail: ISBN@landsbokasafn.is

URL http://www.landsbokasafn.is

India / 978-81 + 978-93

Raja Rammohun Roy National Agency for ISBN

Ministry of Human Resource Development West Block-I, Wing-6, 2nd Floor,

Sector-1, R.K. Puram,

New Delhi-110066

India

Tel (+91-11) 26172903

Tel (+91-11) 26172493/Extn 12

E-mail: anilprabha.isbn@gmail.com

Indonesia / 978-602 + 978-979

Directorate of Legal Deposit

National Library of Indonesia

Tim ISBN/KDT Perpustakaan Nasional RI

Gedung Perpustakaan Nasional RI Blok A

It. 2

Jl. Salemba Raya no. 28A

Jakarta 10430

Indonesia

Tel (+ 62 21) 92920979

Fax (+ 62 21) 3927919

E-mail: isbn.indonesia@gmail.com

URL http://www.pnri.go.id

International NGO Publishers / 978-92

International ISBN Agency

c/o EDItEUR

United House, North Road

London

N7 9DP

United Kingdom

Tel (+44) 2075036418

Fax (+44) 2075036418

E-mail: info@isbn-international.org

URL http://www.isbn-international.org
Iran, Islamic Republic of / 978-600+

978-964

Iran ISBN Agency

Iran Book House

1080 Palestine Crossroad, Enqelab Ave.

PO Box 13145-1455 13156 Tehran

Iran, Islamic Republic of

Tel (+98 21) 66414991

Tel (+98 21) 66496966

Fax (+98 21) 66415360

E-mail: isbn@ketab.org.ir

URL http://www.ketab.ir

Ireland / 978-0 + 978-1

ISBN Agency - UK and Ireland

3rd Floor, Midas House

62 Goldsworth Road

Woking, GU21 6LQ

United Kingdom

Tel (+ 44 (0)) 1483712215

Fax (+ 44 (0)) 1483712214

E-mail: isbn.agency@nielsen.com

URL http://www.isbn.nielsenbook. co.uk

Israel / 978-965

Israeli ISBN Group Agency

Israeli Center for Libraries

Baruch Hirsh 22

POB 801

Bnei Brak 51108

Israel

Tel (+972 3) 6180151

Fax (+972 3) 5798048

E-mail: icl@icl.org.il

Italy / 978-88 + 979-12

EDISER srl

Corso di Porta Romana 108

20122 Milano

Italy

Tel (+39) 0289280805

Fax (+39) 0289280861

E-mail: info@isbn.it

URL http://www.isbn.it

Japan / 978-4

Japan ISBN Agency

c/o Japan Publishers Club Building

6, Fukuro-machi Shinjuku-ku

Tokyo 162-0828

Japan

Tel (+81 3) 32672301

Fax (+81 3) 32672304

E-mail: info@isbn-center.jp

URL http://www.isbn-center.jp
Jordan / 978-9957

ISBN Agency

Department of the National Library

P.O. Box 6070

Amman 11118

Jordan

Tel (+962) (6) 5662845

Tel (+962) (6) 5662854

Fax (+962 6) 5662865

E-mail:nl@nl.gov.jo

URL http://www.nl.gov.jo

Kazakhstan / 978-5 + 978-601 + 978-9965

ISBN agency

National State Book Chamber of the Kazakh Republic

Pushkin Str. 2

Almaty 050016

Kazakhstan

Tel (+7) 7273976204

Fax (+7) 7273976130

E-mail: bookkz@inbox.ru

URL http://www.bookchamber.kz

Kenya / 978-9966

ISBN Agency

Kenya National Library Services

P.O. BOX 30573 - 00100 GPO

Nairobi

Kenya

Tel (+254 20) 2158359

Tel (+254 20) 7786710

Fax (+254 20) 2158352

E-mail: hodnld@knls.ac.ke

URL http://www.knls.or.ke

Korea, Democratic People's Republic of / 978-9946

ISBN DPR Korea Agency

Korea Science and Encyclopedia Publishing House

Secretaire General

Jangyongdong, Sosong District

P.O. Box: 73 Pyongyang

Korea, Democratic People's Republic of

Fax +85023814410

Fax +85023814427

E-mail: isbn-rh@star-co.net.kp

Korea, Republic of / 978-89 + 979-11

Korea ISBN agency

The National Library of Korea

664 Banpo-ro, Banpo-dong, Seocho-Gu

Seoul, 137-702

Korea, Republic of

Tel (+82 2) 5900589

Fax (+82 2) 5900621

E-mail: issnkc@mail.nl.go.kr

URL http://www.nl.go.kr/isbn 
Kosova / 978-86 + 978-9951

Kosova ISBN Agency

National Library of Kosova "Pjetër Bogdani"

Agjencia Kombëtare e ISBN-së

Sheshi "Hasan Prishtina" p.n.

10 000, Prishtinë

Kosova

Tel $(+38138) 212424$

Fax (+38138) 248940

E-mail: bedri.zyberaj@biblioteka-ks. org

URL http://www.biblioteka-ks.org

Kuwait / 978-99906 + 978-99966

ISBN Agency

National Library of Kuwait

P.O.Box. 26182

13122 Safat

Kuwait

Tel (+965) 22929839

Fax (+965) 2415195

E-mail: isbn@nlk.gov.kw

URL http://www.nlk.gov.kw

Kyrgyzstan / 978-9967

ISBN Agency

National Book Chamber

170a, Abdrahmanova street

Bishkek 720040

Kyrgyzstan

Tel (+996 312) 665725

Fax (+996 312) 660377

E-mail: kitep.palatasy@mail.ru

Lao People's Democratic Republic / 978-9932

Lao ISBN Agency

National Library of Laos, Sethathirath Road, P O BOX No 8818, Ministry of Information and Culture

Vientiane

Lao People's Democratic Republic

Tel (+856) 21213029

Fax (+856) 21213029

E-mail: kong_nettavong@hotmail.com

Latvia / 978-5 + 978-9934 + 978-9984

ISBN/ISMN Agency

Latvian National Library

Anglikânu Street 5

Riga, 1050 LV

Latvia

Tel (+371) 67212668

Fax (+371) 67224587

E-mail: isbn@lnb.lv

URL http://www.Inb.lv
Lebanon / 978-614 + 978-9953

ISBN Agency

Ministry of Culture

Marie Curie street, Hamra

Hatab building, 3rd floor

Beirut

Lebanon

Tel (+9611) 744251

Tel (+961 1) 744252

Fax (+961 1) 756305

E-mail: rita_akl@hotmail.com

URL http://www.culture.gov.lb

Lesotho / 978-99911

ISBN Agency

The National University of Lesotho Library

P.O. Roma 180, Maseru

Lesotho

Tel (+266) 22340601

Tel (+266) 28340777

E-mail:wmokotjo@nul.ls

Libya / 978-9959

Libyan ISBN Agency

National Library of Libya

P.O. Box 9127

Benghazi

Libya

Tel (+218 61) 9097073

Tel (+218 61) 9097074

Fax $(+218$ 61) 9097073

Fax (+218 61) 9097074

E-mail: nat_lib_libya@hotmail.com

Lithuania / 978-5 + 978-609 + 978-9955 + 978-9986

ISBN Agency

National Library of Lithuania

Gediminas ave 51

LT-01154 Vilnius

Lithuania

Tel (+370 5) 2398649

Fax (+370 5) 2496055

E-mail: isbnltu@lnb.lt

URL http://www.Inb.lt

Luxembourg / 978-2 + 978-3 + 978-99959

ISBN Agency

Bibliothèque nationale de Luxembourg

37, bd. F.-D. Roosevelt

L-2450 Luxembourg

Luxembourg

Tel (+352) 229755225

Fax (+352) 229755425

E-mail: Charles.Hilger@bnl.etat.lu

URL http://www.bnl.lu
Macao / 978-99937 + 978-99965

Agência do ISBN

Biblioteca Central de Macau

Largo de Sto. Agostinho no. 3

Macau

Macao

Tel (+853) 85986600

Fax (+853) 28314456

E-mail: isbn.bc@icm.gov.mo

URL http://www.library.gov.mo

Macedonia, The Former Yugoslav Republic of / 978-608 + 978-9989

ISBN Agencija na Republika Makedonija

Nacionalna i Univerzitetska Biblioteka

"Sv. Kliment Ohridski"

Bul. Goce Delcev, 6

1000 Skopje

Macedonia, The Former Yugoslav Republic of

Tel + $38923115177 \times 137110$

Fax +38923226846

Fax +38923133418

E-mail: olja@nubsk.edu.mk

URL http://www.nubsk.edu.mk

Malawi / 978-99908 + 978-99960

Malawi National ISBN Agency

National Archives of Malawi

Senior Assistant Librarian

Mkulichi Road, Zomba

P.O. Box 62

Malawi

Tel (+265 1) 525240

Tel $(+265) 997072377$

Fax (+265 1) 525362

E-mail: archivesmalawi@gmail.com

Malaysia / 978-967 + 978-983

ISBN National Centre

National Library of Malaysia

232, Jalan Tun Razak

50572 Kuala Lumpur

Malaysia

Tel (+603) 26814329

Fax (+603) 26811676

E-mail: isbn@pnm.gov.my

URL http://www.pnm.gov.my

Maldives / 978-99915

ISBN Agency

Policy Planning and Research Section, Ministry of Education

9th floor, Velaanaage office Building Ameer Ahmed Magu

Malé

Maldives 
Tel (+960) 3341109

Tel (+960) 3341101

Fax (+960) 3321201

E-mail: fazy@moe.gov.mv

URL http://www.moe.gov.mv

Mali / 978-99952

Agence Nationale de l'ISBN

Direction Nationale des Bibliothèques et de

la Documentation du Mali

B.P. E 4473

Av. Kwamé Nkrumah, ACI 2000

Hamdallaye, Bamako

Mali

Tel (+223) 20299423

Tel (+223) 2236446232

Fax (+223) 20299396

E-mail: dnbd@afribone.net.ml

Malta / 978-99909 + 978-99932 + 978-99957

ISBN Agency for Malta

National Book Council

$\mathrm{C} / \mathrm{o}$, Central Public Library

J.J. Mangion Road

Floriana

Malta

Tel (+356) 21222654

Fax (+356) 21222654

E-mail: joe.debattista@gov.mt

URL http://www.ktieb@org.mt

Mauritius / 978-613 + 978-620 + 978-99903

$+978-99949$

ISBN Agency

Éditions de l'Océan Indien,

22B Marcel Cabon Street, Stanley

Rose Hill

Mauritius

Tel (+230) 4646761

Tel (+230) 4675899

Fax (+230) 4643445

E-mail: eoipublishing@intnet.mu

URL http://www.eoi-info.com

Mexico / 978-607 + 978-968 + 978-970

Agencia ISBN México

INDAUTOR (Instituto Nacional del Derecho de Autor)

Puebla No. 143, piso 2

Col. Roma, Del. Cuauhtémoc

06700 D.F.

Mexico

Tel (+52) 5536018206

Fax (+52) 5536018206

E-mail: rrivera@sep.gob.mx

URL http://www.indautor.sep.gob.mx
Moldova, Republic of / 978-9975

Moldova ISBN Agency

Camera Nationala a Cartii

(National Book Chamber)

Stefan cel Mare 180, of 205

2004, Chisinau

Moldova, Republic of

Tel (+373 22) 295916

Fax (+373 22) 295860

E-mail: ISBN.Moldova@gmail.com

Mongolia / 978-99929 + 978-99962 + 978-99973

ISBN Agency

Mongolian Book Publishers' Association

1st floor, Room 103, Inter Office Building

Amar's Street 2, Sukhbaatar District

P.O. Box-92, Ulaanbaatar 14200

Mongolia

Tel (+976 11) 311023

Fax (+976 11) 327251

E-mail: isbnagency@mbpa.mn

Montenegro / 978-86 + 978-9940

ISBN Agency

Central National Library of Montenegro

"Djurdje Crnojevic"

Bul. Crnogorskih junaka 163

Cetinje 81250

Montenegro

Tel (+382) 41231143

Tel (+382) 41231726

Fax (+382) 41231020

Fax (+382) 41233370

E-mail: cnb@cnb.me

URL http://www.cnb.me

Morocco / 978-9954 + 978-9981

Agence Marocaine de l'ISBN

Bibliothèque Nationale du Royaume du Maroc

Av Ibn Khaldoun, B.P. 1003

Rabat

Morocco

Tel (+212 537) 272300

E-mail: elmoukhi.nawfal@gmail.com

URL http://www.bnrm.ma

Myanmar / 978-99971

ISBN Agency of Myanmar

No 263 National YMCA Building, TheinPhyu

Road, Bothathaung Township, Yangon Myanmar

Tel +951296442

Tel +95 1706082

E-mail:myoaunginnwa@gmail.com
Namibia / 978-0 + 978-99916 + 978-99945

Namibia ISBN Agency

National Library of Namibia

Private Bag 13349, Eugene Marais Street 1

Windhoek

Namibia

Tel (+264 61) 2935305

Fax (+264 61) 2935308

E-mail: Barbara.Jacobi@moe.gov.na

URL http://www.nln.gov.na

Nepal / 978-9937 + 978-99933 + 978-99946

ISBN National Agency

Tribhuvan University

Central Library

Kirtipur

Kathmandu

Nepal

Tel (+977 1) 4331317

Tel (+977 1) 4330834

Fax (+977 1) 4331964

E-mail: tucl@tucl.org.np

URL http://www.tucl.org.np

Netherlands / 978-90 + 978-94

Bureau ISBN

CB

Erasmusweg 10

4104 AK Culemborg

Netherlands

Tel (+31 345) 475855

E-mail: isbn@cb-logistics.nl URL http://www.isbn.nl

New Zealand / 978-0 + 978-1

ISBN Agency

National Library of New Zealand

PO Box 1467

Wellington 6140

New Zealand

Tel (+64 4) 4743074

Fax (+64 4) 4743161

E-mail: ISBN@natlib.govt.nz

URL http://www.natlib.govt.nz

Nicaragua / 978-99924 + 978-99964

Agencia Nicaragüense del ISBN

Biblioteca Nacional de Nicaragua

Palacio Nacional de la Cultura

Frente a Casa Presidencial

Apartado Postal 101

Nicaragua

Tel (+505 2) 222-5088

Fax (+505 2) 222-2722

E-mail: isbnnicaragua@gmail.com

Nigeria / 978-978

Nigerian ISBN Agency

National Bibliographic Control Dept

National Library of Nigeria 
Plot 274 Sanusi-Dantata House

Central Business District, P.M.B. 1, Garki, Abuja.

Nigeria

Tel (+234) 7030617969

E-mail: natbcdnlnig@yahoo.com URL http://www.nln.gov.ng

\section{Norway / 978-82}

The Norwegian ISBN Agency

National Library of Norway

Postboks 2674 Solli

0203 Oslo

Norway

Tel (+47) 75121148

Fax (+47) 75121222

E-mail: isbn-kontoret@nb.no

URL http://www.isbn.no

Oman / 978-99969

Oman ISBN Agency

Ministry of Information

PO Box 600

Muscat 100

Oman

Tel +96824941516

Fax +96824617744

E-mail: abuarwa2272@gmail.com

URL http://www.omanet.om

Pakistan / 978-969

Pakistan ISBN Agency

National Library of Pakistan

Constitution Avenue

Islamabad - 44000

Pakistan

Tel (+92 51) 9202549

Tel (+92 51) 9207456

Fax (+92 51) 9221375

E-mail: ghyour_hussain@hotmail.com URL http://www.nlp.gov.pk

Palestine, State of / 978-9950

Palestine ISBN Agency

P.O.Box 3523

Ramallah

Palestine, State of

Tel (+970-2) 2950618

Fax (+970 2) 2950617

E-mail: info@isbn.ps

URL http://www.isbn.ps

Panama / 978-9962

Agencia Panameña del ISBN

Biblioteca Nacional "Ernesto J. Castillero R"

San Francisco, Vía Porras

Parque Recreativo y Cultural Omar

Apartado Postal 7906 Zona 9, Panamá

Panama
Tel (+507) 2218360

Tel (+507) 2215965

Fax (+507) 2215965

E-mail: grivera@binal.ac.pa

URL http://www.binal.ac.pa

Papua New Guinea / 978-9980

Papua New Guinea ISBN Agency

Technical Services Librarian

National Library of PNG

P.O. Box 734

Waigani, N.C.D.

Papua New Guinea

Tel (+675) 77014853

Fax (+675) 3254251

E-mail:chris_meti@education.gov.pg

Paraguay / 978-99925 + 978-99953 + 978-99967

Agencia ISBN Paraguay

Secretaría Nacional de Cultura

Capitán Figari № 898 esquina Manuel Domínguez

Asunción

Paraguay

Tel (+595) 921223465
Fax (+595) 921230001
E-mail: isbn@cultura.gov.py
URL http://www.cultura.gov.py

Peru / 978-612 + 978-9972

Agencia Peruana del ISBN

Biblioteca Nacional del Perú

Directora General del Centro Bibliográfico

Av. de la Poesía 160

San Borja, Lima 41

Peru

Tel (+511) $5136900 \times 71367110$

Fax (+511) 5137060

E-mail: isbn.bnp@gmail.com

URL http://www.bnp.gob.pe/

Philippines / 978-621 + 978-971

Standard Book Numbering Agency

The National Library of the Philippines

TM Kalaw St

P.0 Box 2926

Ermita Manila 1000

Philippines

Tel (+ 63 2) 3105032

Tel (+ 63 2) 3367200

Fax (+ 632$) 5242329$

E-mail: szamora56@yahoo.com

URL http://web.nlp.gov.ph/nlp/

Poland / 978-83

Krajowe Biuro ISBN

Biblioteka Narodowa

Al. Niepodległości 213
02-086 Warszawa

Poland

Tel (+48 22) 6082410

Tel (+48 22) 6082432

Fax (+48 22) 6082974

Fax (+48 22) 8255729

E-mail: bnisbn@bn.org.pl

URL http://www.bn.org.pl

Portugal / 978-972 + 978-989

ISBN Agency

Associação Portuguesa de Editores e

Livreiros

Largo do Andaluz, 16, 1.․․, esq.

P - 1050-004 Lisboa

Portugal

Tel (+351 21) 8473591

Fax (+351 21) 8489377

E-mail: isbn@apel.pt

URL http://www.apel.pt

Qatar / 978-9927 + 978-99921

ISBN group agency

National Library

P.O. Box 205

Doha

Qatar

Tel (+974) 44389888

Fax (+974) 44412308

E-mail: alansari_123@hotmail.com

Romania / 978-606 + 978-973

Centrul National ISBN-ISSN-CIP

Biblioteca Nationala a României

Bd. Unirii nr. 22, sector 3

030833 Bucuresti

Romania
Tel (+40 21) 3112635
Fax (+40 21) 3124990
E-mail: isbn@bibnat.ro
URL http://www.bibnat.ro

Russian Federation / 978-5

Russian ISBN Agency

Russian Book Chamber

Kremlevskaja nab. 1/9

Moscow 119019

Russian Federation

Tel (+7 495) 6975608

Tel (+7 495) 6889689

Fax (+7 499) 7660090

Fax (+7 495) 6889689

E-mail: info@bookchamber.ru, isbn@ bookchamber.ru

URL http://www.bookchamber.ru 
Saudi Arabia / 978-603 + 978-9960 Book Registration \& Numbering Dept King Fahd National Library

P.O. Box 7572

Riyadh 11472

Saudi Arabia

Tel (+966 1) 4624888

Fax (+966 1) 4645341

E-mail: KFNL-ISN@kfnl.gov.sa

URL http://www.kfnl.gov.sa

Serbia / 978-86

ISBN Agency

National Library of Serbia

Skerliceva 1

11000 Belgrade

Serbia

Tel (+381 11) 2459444

Tel (+381 11) 2451242

Fax (+381 11) 2459444

E-mail: emilija.brasic@nb.rs

URL http://www.nb.rs

Seychelles / 978-99931

ISBN Agency

Ministry of Local Government Sports \& Culture

National Library

Victoria

P.O. Box 45, Mahé

Seychelles

Tel (+248) 321333

Fax (+248) 323183

E-mail:marylynhen@yahoo.com

Sierra Leone / 978-99910

ISBN Agency

Sierra Leone Library Board

Rokel Street

Freetown

Sierra Leone

Tel (+232) 22226993

Tel (+232) 76680545

E-mail:sielib2002@yahoo.com

Singapore / 978-981 + 978-9971

ISBN Agency

National Library Board, Library Supply Centre

3 Changi South St 2 \#02-00

Xilin Districentre Building $B$

486548

Singapore

Tel (+65) 65467271

Fax (+65) 65467262

E-mail: legaldeposit@library.nlb.gov.sg

URL http://deposit.nl.sg
Slovakia / 978-80

Slovak ISBN Group Agency

Slovak National Library

Nám J.C. Hronského 1

03601 Martin

Slovakia

Tel (+421 43) 2451140

Tel (+421 43) 2451138

Fax (+421 43) 2451505

E-mail: isbn@snk.sk

URL http://www.snk.sk

Slovenia / 978-86 + 978-961

ISBN Agency

National and University Library

P.p. 259

1001 Ljubljana

Slovenia

Tel (+386 1) 5861333

Fax (+386 1) 5861311

E-mail: isbn@nuk.uni-li.si

URL http://www.nuk.uni-lj.si

South Africa / 978-0 + 978-1

ISBN Agency

The National Library of South Africa

P.O. Box 397

Pretoria 0001

South Africa

Tel (+27 (0)12) 4019718

Fax (+27 (0)12) 3242441

E-mail: ISN.agency@nlsa.ac.za

South Pacific / 978-982

Regional ISBN Centre

The University of the South Pacific Library

Private Mail bag

Laucala Bay Road

Suva

Fiji

Tel (+679) 3232200

Tel (+679) 3232251

Fax (+679) 3231528

E-mail: chambers_v@usp.ac.fj, sjoan. yee@usp.ac.fj

URL http://www.usp.ac.fj

Spain / 978-84

Agencia del ISBN

Cea Bermúdez, 44, 2 derecha

28003 Madrid

Spain

Tel (34) 902105389

E-mail:agencia@agenciaisbn.es

URL http://agenciaisbn.es
Sri Lanka / 978-955

ISBN Agency

National Library and Documentation Services Board

No. 14, Independence Avenue

Colombo 07

Sri Lanka

Tel (+94 1) 12687581

Tel (+94 1) 12685198

Fax (+94 1) 12685201

Fax (+94 1) 12687583

E-mail: ch@mail.natlib.lk

URL http://www.natlib.lk

Srpska, Republic of / 978-86 + 978-99938+ 978-99955 + 978-99976

ISBN Agency

National and University Library of Republic of Srpska

Jevrejska 30

78000 Banja Luka

Bosnia and Herzegovina

Tel (+ 387 51) 215 859/ext. 124

Fax (+ 387 51) 217040

E-mail:marija.pavlovic@nub.rs

URL http://www.nub.rs

Sudan / 978-99942

ISBN Agency

The National Library

Al-Amarat St 9 Building no 9

P.O. Box 6279

Khartoum

Sudan

Tel (+249) 183560969

Fax (+249) 183560951

E-mail: natlib10@hotmail.com

URL http://www.natlib.gov.sd

Suriname / 978-99914

Standard Book Numbering Agency

Publishers Association Suriname

Domineestraat 32 boven

P.O. Box 1841

Paramaribo

Suriname

Tel (+597) 472545

Fax (+597) 410563

E-mail: postmaster@interfundgroup. com

Swaziland / 978-0 + 978-1

ISBN Agency

University of Swaziland Libraries

Special Collections

Private Bag 4

Kwaluseni

Swaziland 
Tel (+268) 5184011 ext 2399 or 2298

Fax (+268) 5185276

E-mail: abahle@uniswacc.uniswa.sz URL http://www.library.uniswa.sz

Sweden / 978-91

Swedish ISBN Agency

National Library of Sweden

National Bibliography

Box 5039

10241 Stockholm

Sweden

Tel (+46) 107093367

Tel (+46) 107093090

Fax (+46) 107093925

E-mail: isbn@kb.se

URL http://www.kb.se/isbn

Switzerland / 978-2 + 978-3

ISBN-Agentur Schweiz

c/o Schweizer Buchhändler- und Verleger-

Verband SBVV

Alderstrasse 40

Postfach

8034 Zürich

Switzerland

Tel (+41 44) 4213601

Fax (+41 44) 4213618

E-mail: isbn@sbvv.ch

URL http://www.sbvv.ch

Syrian Arab Republic / 978-9933

Syrian ISBN Agency

The Syrian Publishers Association

P O Box 5888

Halbouni, Damascus

Syrian Arab Republic

Tel (+963) 112246636

Tel (+963) 112246612

Fax (+963) 112246657

E-mail: info@syrianpublishers.com URL http://www.syrianpublishers.com

Taiwan / 978-957 + 978-986

ISBN Agency

National Central Library

20 Chung Shan South Road

Taipei 10001

Taiwan

Tel (+886 2) $23619132 \times 721$

Fax (+886 2) 23316515

E-mail: isbn@ncl.edu.tw

URL http://isbn.ncl.edu.tw

Tajikistan / 978-99947 + 978-99975

ISBN Agency

House of Book of Tajikistan

N Karoboev Ave 17

Dushanbe City

Tajikistan
Tel (+992) 372337381

Tel (+992) 37935069137

E-mail: isbntajikistan@mail.ru

Tanzania, United Republic of / 978-9976 + 978-9987

National Bibliographic Agency

Director General

Tanzania Library Services Board

P.O. Box 9283

Dar es Salaam

Tanzania, United Republic of

Tel (+255 22) 2150048

Tel (+255 22) 2150049

Fax (+255 22) 2151100

E-mail: innosenciaj@gmail.com

Thailand / 978-611 + 978-616 + 978-974 ISBN Agency

National Library of Thailand

Library Resource Development Group

Samsen Road, Dusit

Bangkok 10300

Thailand

$$
\begin{aligned}
& \text { Tel +66 (0) } 2823807 \\
& \text { Tel +66 (0) } 28238089 \\
& \text { Fax +66 (0) } 2815450 \\
& \text { E-mail: isbn@nlt.go.th } \\
& \text { URL http://www.nlt.go.th }
\end{aligned}
$$

Tunisia / 978-9938 + 978-9973

National ISBN Agency

Bibliothèque Nationale de Tunisie

Service de la Documentation et de

l'information

Rue 3 Sept 1934 no 16

1006 Tunis, BP 42 TUNIS RP 1000

Tunisia

Tel (+216) 71572706

Tel (+216) 71573303

Fax (+216) 71572887

E-mail: lotfi.ziedi@bnt.nat.tn

URL http://www.Bibliotheque.nat.tn

Turkey / 978-605 + 978-975 + 978-9944 ISBN Türkiye Ajansý

Kültür ve Turizm Bakanligi

Fevzipaşa Mah., Cumhuriyet Bulvarı

No:4 B Blok Kat 1248 Ulus

Ankara

Turkey

Tel (+90 312) $3099050-60$

Fax (+90 312) 3098999

E-mail: isbn@kultur.gov.tr

URL http://www.isbnturkiye.gov.tr

Uganda / 978-9970

ISBN Agency

National Library of Uganda

Plot 50, Buganda Road
P.0. Box 4262

Kampala

Uganda

$$
\begin{aligned}
& \text { Tel }+256414233633 \\
& \text { Tel }+256414254661 \\
& \text { Fax +256 } 114348625 \\
& \text { E-mail: admin@nlu.go.ug } \\
& \text { URL http://www.nlu.go.ug }
\end{aligned}
$$

Ukraine / 978-5 + 978-617 + 978-966

National ISBN Agency

Derzhavna naukova ustanova "Knyzhkova Palata Ukrainy imeni Ivana Federova"

27, Yuri Gagarin Ave \#101

Kyiv 02660

Ukraine

Tel (+380 044) 292-01-34

Tel (+380 044) 5735236

Fax (+380 044) 5735236

E-mail: isbn@ukrbook.net

URL http://www.ukrbook.net

United Arab Emirates / 978-9948

Ministry of Information and Culture - Copyright Section - ISBN Agency

P.O. Box 17

Abu Dhabi

United Arab Emirates

Tel (+971) 24083463

Tel (+971) 24083580

Fax (+971) 24466127

Fax (+971) 24433672

E-mail: basema.m.younes@gmail.com

United Kingdom / 978-0 + 978-1

Nielsen Book - ISBN Agency

Midas House, 3rd Floor

62 Goldsworth Road

Woking

Surrey GU21 6LQ

United Kingdom

Tel +44 (0) 1483712215

Fax +44 (0) 1483712214

E-mail: isbn.agency@nielsen.com

URL http://www.isbn.nielsenbook. co.uk

United States / 978-0 + 978-1

R.R. Bowker, LLC - US ISBN Agency

630 Central Ave.

New Providence, NJ 07974

United States

Tel $(+1)$ 877-310-7333

Tel (+1) 888-2695372

Fax (+1 908) 219-0188

E-mail: isbn-san@bowker.com

URL http://www.isbn.org 
Uruguay / 978-9974

ISBN Agency

Biblioteca Nacional

Sección Bibliografía Nacional - Agencia ISBN

18 de Julio 1790

C.P. 11200 Montevideo

Uruguay

Tel (+598 2) 409-60-11

Tel (+598 2) 409-60-13 x 216

Fax (+598 2) 4016716

E-mail: isbnuy@adinet.com.uy

URL http://www.bibna.gub.uy

Uzbekistan / 978-9943

National Library of Uzbekistan - ISBN Agency

National Library of Uzbekistan

Navoi street, 1

Tashkent, 100017

Uzbekistan

Tel +998 712328391

Tel +998712394440

Fax +998712391658

E-mail: navoi@natlib.uz

URL http://www.natlib.uz
Venezuela, Bolivarian Republic of / 978-980

Agencia Venezolana del ISBN

CENAL, Centro Simón Bolívar

Torre Norte, Piso 20, El Silencio

Caracas 1011

Venezuela, Bolivarian Republic of

Tel (+58 212) 4822020

Tel (+58 212) 5765370

Fax (+58-212) 4822020

E-mail: cenal.isbnvenezuela@gmail. com

Viet Nam / 978-604

Vietnam ISBN Agency - Publishing Department

Ministry of Information and Communication 10 Duong Thanh, Hoan Kiem

Hanoi

Viet Nam

Tel +84438285641 (ext 304)

Tel +84906827876

E-mail: thuynga0908@gmail.com
Zambia / 978-9982

The ISBN Secretariat

Booksellers \& Publishers Association of Zambia

P.O. Box 51109

Lusaka

Zambia

Tel (+260) 977746477

Tel (+260 1) 253952

E-mail: dongo.banda@gmail.com

Zimbabwe / 978-0 + 978-1

National Archives of Zimbabwe

Causeway, Private Bag 7729

Harare

Zimbabwe

Tel (+263 4) 792741

Fax (+263 4) 792398

E-mail: archives@archives.gov.zw 